\title{
Intervención educativa para disminuir la prevalencia del consumo de alcohol en escolares de la I.E. Santa Isabel
}

\author{
Educational intervention to reduce the prevalence of alcohol consumption in schoolchildren of Santa Isabel \\ Educational Institution
}

Vilma Amparo Junchaya Yllescas ${ }^{1, \mathrm{a}}$, Diana Esmeralda Andamayo Flores ${ }^{1, \mathrm{~b}}$,
Renee Soledad Orrego Cabanillas ${ }^{1, \mathrm{a}}$, Martha Raquel Valderrama Sueldo ${ }^{1, \mathrm{a}}$

RESUMEN

Objetivos: Evaluar el efecto de la intervención educativa que permita disminuir la prevalencia del consumo de alcohol en escolares de la I.E. Santa Isabel. Material y Métodos: Se emplearon los métodos basados en la participación, responsabilidad y técnicas directas por medio del lenguaje hablado y materiales audiovisuales, incluyendo charlas, consejerías y lluvias de ideas etc. (folletos y trípticos), empleándose siete sesiones de clase de intervención educativa de una hora. El tipo y nivel de investigación fue aplicada, de diseño cuasi-experimental con dos grupos, uno control y el otro experimento, con estudio pretest postest, realizado en una población de 418 (19 aulas) y una muestra de 204 estudiantes divididos en dos grupos, la técnica utilizada fue la encuesta y la intervención educativa, siendo el instrumento un cuestionario. Resultados: El 38\% de los escolares de la IE Santa Isabel consumen alcohol, mientras que un $62 \%$ de ellos no lo hacen, la frecuencia del consumo es más de 2 veces al mes en $51 \%$; siendo los factores del consumo, cuando un $31 \%$ de los escolares lo hacen por diversión, seguido un $21 \%$ porque les gusta, un $36 \%$ de los escolares sostienen que consumen alcohol porque han faltado con claridad a las reglas que comunican los padres, un $79 \%$ porque algún miembro de la familia ha consumido alcohol y el $62 \%$ porque en la mayoría de las fiestas no hay supervisión de adultos. Conclusiones: El efecto de la intervención educativa permitió disminuir la prevalencia del consumo de alcohol en escolares de La I.E. Santa Isabel significativamente y consideran que su consumo es malo en el grupo experimento a diferencia del grupo control.

PALABRAS CLAVE: Intervención educativa, prevalencia, consumo de alcohol, escolares.

\section{SUMMARY}

Objectives: Evaluate the effect of the educational intervention that allows to reduce the prevalence of alcohol consumption in schoolchildren of I.E. Santa Isabel. Material and Methods: We used the methods based on participation, responsibility and direct techniques by through words and audiovisuals including lectures, counseling and brainstorming etc (leaflets and triptychs), using seven sessions class of One-hour educational intervention. The type and level of research was applied, of quasi-experimental design with two groups, one control and the other experiment, with pretest-posttest study, conducted in a population of 418 ( 19 classrooms) and a sample of 204 students divided in two. groups, the technique used was the survey and the educational intervention, being the instrument a questionnaire. Results: The 38 percent of IE Santa Isabel students consume alcohol, while 62 percent of them don't, the frequency of consumption is more than 2 times a month in 51 percent; being the factors of consumption, when $31 \%$ schoolchildren do it for fun, followed by $21 \%$ because they like it, $36 \%$ of the schoolchildren maintain that they

Universidad Privada de Huancayo Franklin Roosevelt. Huancayo, Perú.

Magister; ${ }^{\mathrm{b}}$ Doctora. 
consume alcohol because they have clearly lacked the rules communicated by parents, a $79 \%$ because some family member has consumed alcohol and the $62 \%$ because in majority of the parties there is no supervision of adults. Conclusions: The effect of the educational intervention allowed to decrease the prevalence of alcohol consumption in schoolchildren of I.E Santa Isabel significantly in the experimental group, unlike the control group.

KEYWORDS: Educational intervention, prevalence, alcohol consumption, school.

\section{INTRODUCCIÓN}

Los adolescentes y estudiantes universitarios corren un alto riesgo de iniciarse en el consumo de alcohol y existe un creciente cuerpo de literatura sobre los efectos cognitivos neurotóxicos y dañinos que este produce en jóvenes que toman su primer trago a la edad de 12 años. Además, los episodios excesivos de consumo compulsivo o excesivo de alcohol afectan los hábitos de estudio y erosionan el desarrollo de habilidades de transición hacia la edad adulta (1).

Así también la publicidad genera una actitud positiva hacia el alcohol que hace olvidar ser una droga extremadamente peligrosa que usada sin moderación puede destruir por completo a sus consumidores, muy sensibles, especialmente en el caso de los adolescentes. Así también según estudios de los anuncios televisivos de alcohol "reproducen los hábitos y modos de ingesta juveniles (los bares, la calle, la música, el grupo de amigos)". En ellos "se hace más hincapié en los valores simbólicos que en los valores de uso; es decir, se habla más del prototipo del bebedor que de la bebida en sí, haciendo hincapié en el significado del alcohol como indicador de pertenencia a un grupo de referencia" $(2$, $3,4)$.

La adolescencia es la etapa de la vida de paso de la infancia a la edad adulta del ser humano y constituye un período transicional, en esta etapa se produce el período idóneo para la adopción de hábitos y actitudes, especialmente en el uso de sustancias adictivas que posteriormente pueden permanecer en la adultez, si no son bien manejadas, y llegan a afectar la salud del individuo y la sociedad, por lo que estudios previos indican que el tabaquismo y el consumo de alcohol se inician en los años de adolescencia como resultado de la interacción de factores sociales, culturales e interpersonales.

Cuando la intervención educativa es un proceso que desemboca en la realización de los aprendizajes que son efectivamente integrados en la estructura cognitiva de la persona que aprende, con lo que se asegura su memorización comprensiva y su funcionalidad; por ello la intervención realizada en la IE. Santa Isabel, estuvo enmarcado como propuesta para instruir a los adolescentes sobre el peligro de esta sustancia y beber de manera que no dañen la salud o a mantener la abstinencia (5).

Por tales razones se realizó esta investigación con la finalidad de contribuir a la reducción de la demanda del consumo de bebidas alcohólicas en adolescentes.

\section{MATERIAL Y MÉTODOS}

Esta investigación corresponde a un estudio de tipo aplicada, que se caracterizó por la implementación de la intervención educativa en escolares de la I.E. Santa Isabel con la finalidad de disminuir la prevalencia del consumo de alcohol.

La presente investigación empleó un diseño cuasiexperimental con dos grupos, uno control y el otro experimental, con estudio pretest-postest.

El diagrama representativo que se ha establecido es el siguiente.

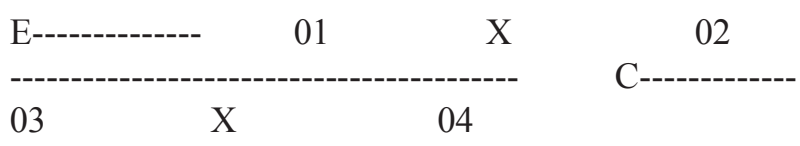

Donde:

$\mathrm{E}=$ Grupo experimental

$\mathrm{C}=$ Grupo de control

$\mathrm{X}=$ Tratamiento experimental

0102 = Observación de la intervención educativa de las sesiones en escolares para evaluar prevalencia, como grupo experimento.

0304 = Observación del comportamiento de los escolares, como grupo control para evaluar prevalencia La población del estudio, estuvo constituida por todos los escolares de tercer año de secundaria de la I.E. Santa Isabel que se encuentran matriculados y corresponde a 418 (19 aulas).

La muestra la conforman 204 escolares de la I.E. 
Santa Isabel y para determinar el tamaño de la muestra aleatoria se utilizó una muestra representativa y de la siguiente manera:

1. Se elaboró un listado de todos los adolescentes de secundaria de la I.E. Santa Isabel.

2. Se generó los números aleatorios de acuerdo al tamaño de la muestra(n) cuyos valores se encontraron entre $1 \mathrm{y} \mathrm{n}$.

3. Se elaboró un listado de la muestra con todos los estudiantes que fueron seleccionados aleatoriamente.

La técnica utilizada fue la encuesta y la intervención educativa con sesiones de clase una vez por semana y se cumplió de acuerdo al horario de tutoría establecido por la I.E. Santa Isabel, empleándose como instrumento un cuestionario para la recolección de los datos estructurado por 5 preguntas, dirigido a los escolares adolescentes que accedieron a participar en la investigación, y la información fue manejada sólo por los investigadores, el cual fue aplicado antes y después de la intervención educativa de 8 sesiones de clase. El instrumento, se encontraba estructurado con una presentación e instructivo para el llenado correcto por los escolares, y de acuerdo a las dimensiones de la investigación.

\section{Técnicas de procesamiento de los datos.}

El estudio constó de 3 etapas: diagnóstica, de intervención educativa y de evaluación. Una vez concluida la intervención educativa con las sesiones de clase por periodo de 3 meses, se aplicó nuevamente el instrumento utilizado en la etapa diagnóstica y se evaluó el impacto de los resultados posteriores a las acciones educativas.

Para el cumplimiento del análisis estadístico de datos, se utilizó el SPS. Una vez elaborado esta matriz de hoja de cálculo, se construyeron tablas de frecuencia y figuras de barras en las cuales se muestra la prevalencia del consumo de alcohol antes y después de la intervención educativa en la I.E. Santa Isabel. Luego se realizó un análisis bivariado para identificar la asociación entre la intervención educativa y la prevalencia del consumo de alcohol.

Enseguida las tablas y figuras fueron interpretadas a profundidad para explicar el porqué de los resultados obtenido en cada medición, los mismos que serán utilizados en la discusión de esta investigación y almacenándose los datos en el PASW Statistic vers. 18.0 .

\section{RESULTADOS}

Una vez procesada y analizada la información se obtuvieron los siguientes resultados:

En la tabla 1 y gráfico 1, se aprecia que el $38 \%$ de los escolares de la IE Santa Isabel consumen alcohol, mientras que un $62 \%$ de ellos no lo hacen.

Tabla 1. Prevalencia del consumo de alcohol en la muestra investigada de escolares de la IE. Santa Isabel.

\begin{tabular}{lcc}
\hline PREVALENCIA & $\mathbf{n}$ & $\mathbf{\%}$ \\
\hline $\mathrm{Si}$ & 77 & 38 \\
$\mathrm{No}$ & 127 & 62 \\
TOTAL & 204 & 100 \\
\hline
\end{tabular}

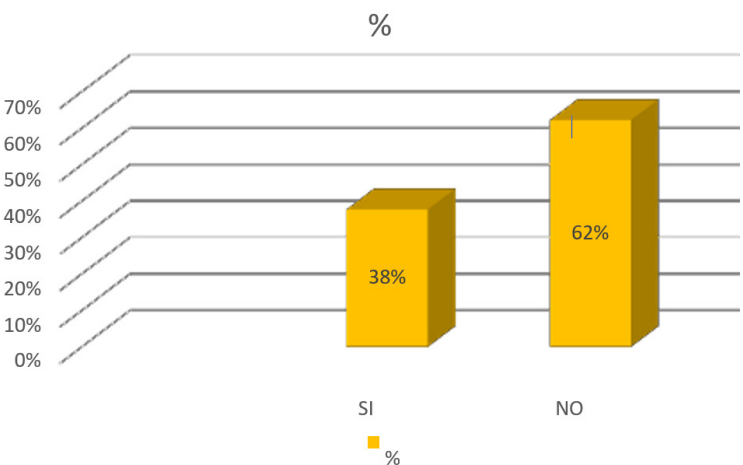

Gráfico 1. Prevalencia del consumo de alcohol en la muestra investigada de escolares de la IE. Santa Isabel

Tabla 2. Frecuencia del consumo de alcohol en escolares de la IE. Santa Isabel.

\begin{tabular}{lc}
\hline \multicolumn{1}{c}{ CATEGORIA } & n \\
\hline Más de 2 veces al mes & 39 \\
1 o 2 veces al mes & 18 \\
Más de 2 veces a la semana & 15 \\
1 a 2 veces por semana & 5 \\
TOTAL & 77 \\
\hline
\end{tabular}

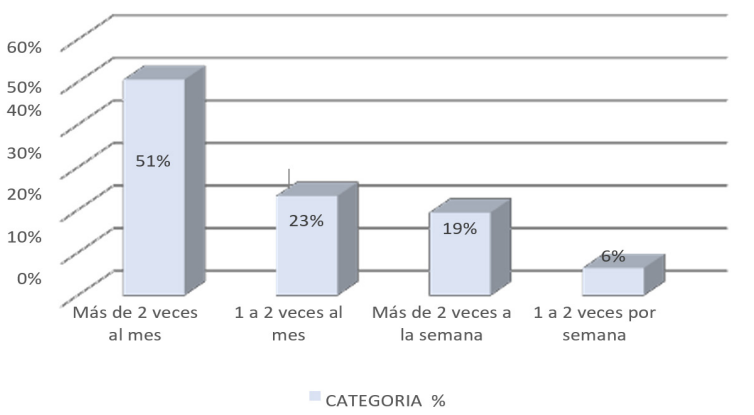

Gráfico 2. Frecuencia del consumo de alcohol en escolares de la IE. Santa Isabel. 
En la tabla 2 y gráfico 2, se aprecia que el $51 \%$ de los escolares respondieron que consumen la bebida alcohólica más de 2 veces al mes, seguido de 1 a 2 veces en el $23 \%$.

En la tabla 3 y figura 3, se presenta que el $35 \%$ de los escolares respondieron que consumen alcohol por diversión, seguido un $18 \%$ porque les gusta.

La tabla 3 y gráfico 4 , presenta que el $36 \%$ de los escolares sostienen que consumen alcohol porque han faltado con claridad en las reglas que comunican los padres, seguido un $31 \%$ por discusiones de los padres, un $30 \%$ porque algún miembro de la familia ha consumido alcohol.

Tabla 3. Distribución por factores de riesgo personales que conllevan al consumo de bebidas alcohólicas en escolares de la IE Santa Isabel.

\begin{tabular}{lcc}
\hline FACTORES PERSONALES & $\mathbf{n}$ & $\mathbf{\%}$ \\
\hline Porque si & 8 & 10 \\
Porque lo hacen los demás & 1 & 1 \\
Para relajarme & 6 & 8 \\
Porque me gusta & 16 & 21 \\
Por timidez & 5 & 6 \\
Por divertirme & 27 & 35 \\
Para olvidar & 14 & 18 \\
Para relacionarme con los demás & 0 & 0 \\
TOTAL & 77 & 100 \\
\hline
\end{tabular}

En la tabla 5 y gráfico 5, se aprecia que un $79 \%$ de los escolares sostienen que los factores que han ocasionado el consumo de alcohol es que algún miembro de la familia ha consumido alcohol, seguido de un $21 \%$ lo hacen porque alguno de los amigos le ha regalado el alcohol.

En la tabla 6y gráfico 6, se puede observar que el $62 \%$ de los escolares respondieron que consumen el alcohol por factores que la mayoría acuden a fiestas no supervisados por adultos, mientras que un 38\% sostienen que lo consumen para sentirse bien.

En la tabla 7, se puede apreciar siete sesiones de clase que fueron otorgadas a los escolares de la IE. Santa Isabel como grupo experimento, empleándose diapositivas y proyector multimedia para su exposición.

En la tabla 8 y figura 8, se aprecia en el GE, que de una prevalencia del consumo de 36 antes de la intervención educativa este disminuye significativa en su respuesta a 6, a diferencia que en el GC de 41 escolares que respondieron que consumían alcohol, como post test respondieron 38, no existiendo variación significativa.

En la tabla 9 y gráfico 9 , se puede apreciar que un $50 \%$ de los estudiantes del GE, responden que el consumo de alcohol afecta la capacidad de razonamiento y juicio, un 19\% sostiene que produce un daño permanente de la memoria y un $17 \%$ refiere que produce relaciones sociales de alto riesgo, en cambio en el GC, solo un $2 \%$ respondieron que el consumo de alcohol afecta la capacidad de razonamiento y juicio,

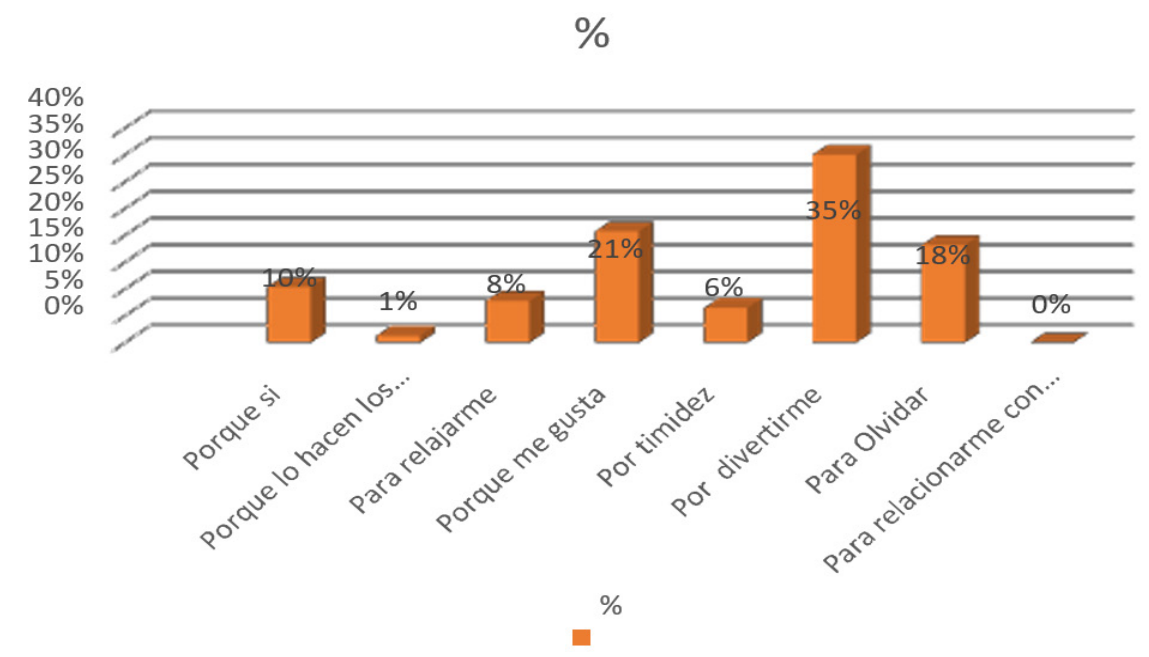

Gráfico 3. Distribución por factores de riesgo personales que conllevan al consumo de bebidas alcohólicas en escolares de la IE Santa Isabel. 
Tabla 4. Distribución por factores de riesgo familiares que conllevan al consumo de bebidas alcohólicas en escolares de la IE Santa Isabel.

\begin{tabular}{lcc}
\hline \multicolumn{1}{c}{ FACTORES FAMILIARES } & $\mathbf{n}$ & $\mathbf{\%}$ \\
\hline $\begin{array}{l}\text { Algún miembro de la familia } \\
\text { ha consumido alcohol }\end{array}$ & 23 & 30 \\
$\begin{array}{l}\text { Ha faltado claridad en las reglas } \\
\text { que comunican los padres }\end{array}$ & 28 & 36 \\
$\begin{array}{l}\text { Discusiones frecuentes entre } \\
\text { los padres }\end{array}$ & 24 & 31 \\
$\begin{array}{l}\text { Con frecuencia mis padres } \\
\text { desconocen donde me encuentro }\end{array}$ & 2 & 3 \\
TOTAL & 77 & 100 \\
\hline
\end{tabular}

Tabla 5. Distribución por factores de riesgo amicales que conllevan al consumo de bebidas alcohólicas en escolares de la IE Santa Isabel.

\begin{tabular}{lcc}
\hline $\begin{array}{l}\text { FACTORES AMICALES/ } \\
\text { CONTEXTO }\end{array}$ & n & \% \\
\hline $\begin{array}{l}\text { Algùn miembro de la familia } \\
\text { ha consumido alcohol }\end{array}$ & 61 & 79 \\
$\begin{array}{l}\text { Alguno de los amigos le ha regalado } \\
\text { alcohol }\end{array}$ & 16 & 21 \\
TOTAL & & \\
\hline
\end{tabular}

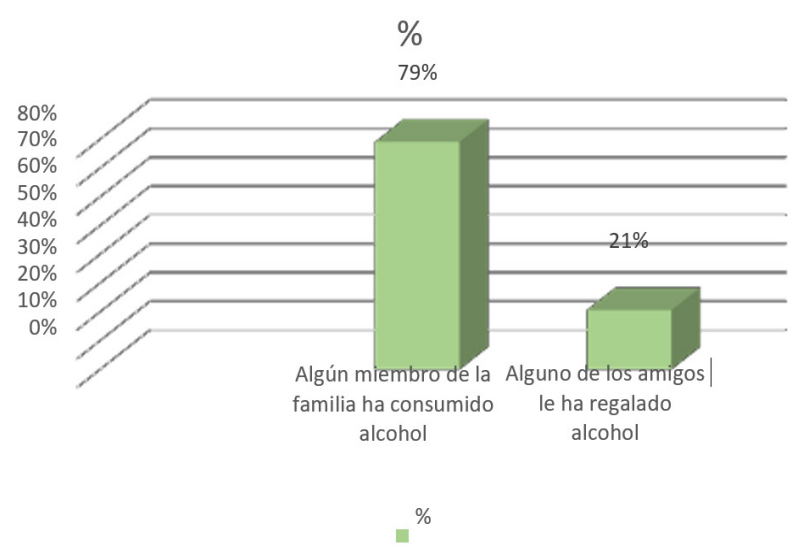

Fuente: Elaboración propia - julio 2018

Gráfico 5. Distribución por factores de riesgo amicales que conllevan al consumo de bebidas alcohólicas en escolares de la IE Santa Isabel.
Tabla 6. Distribución por factores de riesgo sociales que conllevan al consumo de bebidas alcohólicas en escolares de la IE Santa Isabel.

\begin{tabular}{lcc}
\hline FACTORES AMICALES/CONTEXTO & n & $\%$ \\
\hline $\begin{array}{l}\text { En la mayoría de fiestas donde se } \\
\text { encuentra }\end{array}$ & 48 & 62 \\
no hay supervisión de adultos & & \\
Se consume alcohol para sentirse bien & 29 & 38 \\
TOTAL & 77 & 100 \\
\hline
\end{tabular}

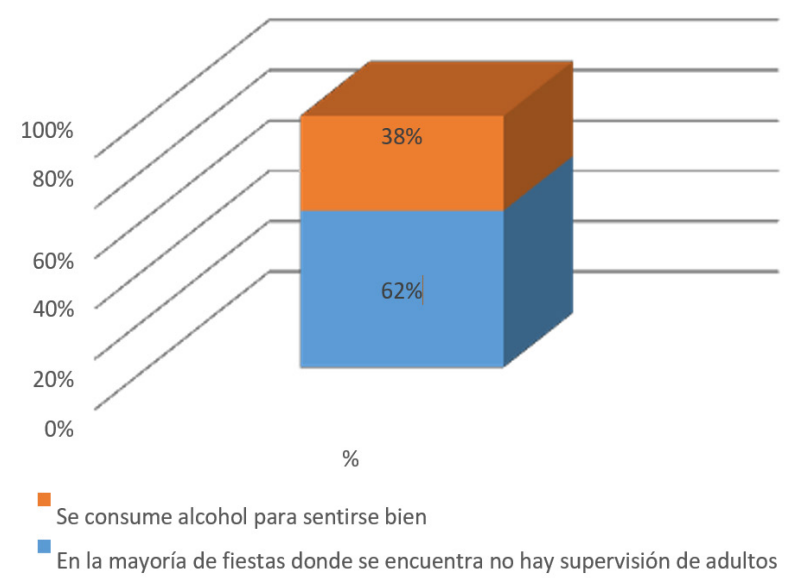

Fuente: Tabla 6

Gráfico 6. Distribución por factores de riesgo sociales que conllevan al consumo de bebidas alcohólicas en escolares de la IE Santa Isabel.

Tabla 7. Intervención educativa con sesiones de clase al grupo experimento.

\begin{tabular}{cl}
$\begin{array}{c}\text { NÚMERO } \\
\text { DE } \\
\text { SESIÓN }\end{array}$ & \multicolumn{1}{c}{ ACTIVIDAD } \\
\hline 1 & $\begin{array}{l}\text { Aplicación del pretest } \\
\text { Introducción al consumo de bebidas } \\
\text { alcohólicas }\end{array}$ \\
3 & $\begin{array}{l}\text { Taller: Mi familia y yo } \\
\text { Taller: El alcohol en tu cuerpo y lo } \\
\text { que ocasiona } \\
4\end{array}$ \\
5 & $\begin{array}{l}\text { Taller: Factores o cusas del consumo } \\
\text { de alcohol en adolescentes } \\
\text { Taller: Tratamiento del consumo de } \\
\text { alcohol en adolescentes } \\
\text { Evaluación post test: Feedback y } \\
\text { clausura de la intervención educativa }\end{array}$
\end{tabular}


Tabla 8. Prevalencia del consumo de alcohol en escolares de la IE. Santa Isabel antes de la intervención educativa distribuidos como Grupo Experimento y Grupo Control.

\begin{tabular}{|c|c|c|c|c|c|c|c|}
\hline \multicolumn{4}{|c|}{ GRUPO EXPERIMENTO } & \multicolumn{4}{|c|}{ GRUPO CONTROL } \\
\hline \multicolumn{2}{|c|}{ ANTES } & \multicolumn{2}{|c|}{ DESPUES } & \multicolumn{2}{|c|}{ ANTES } & \multicolumn{2}{|c|}{ DESPUES } \\
\hline $\mathrm{Si}$ & No & $\mathrm{Si}$ & No & $\mathrm{Si}$ & No & $\mathrm{Si}$ & No \\
\hline 36 & 66 & 6 & 96 & 41 & 61 & 38 & 64 \\
\hline
\end{tabular}

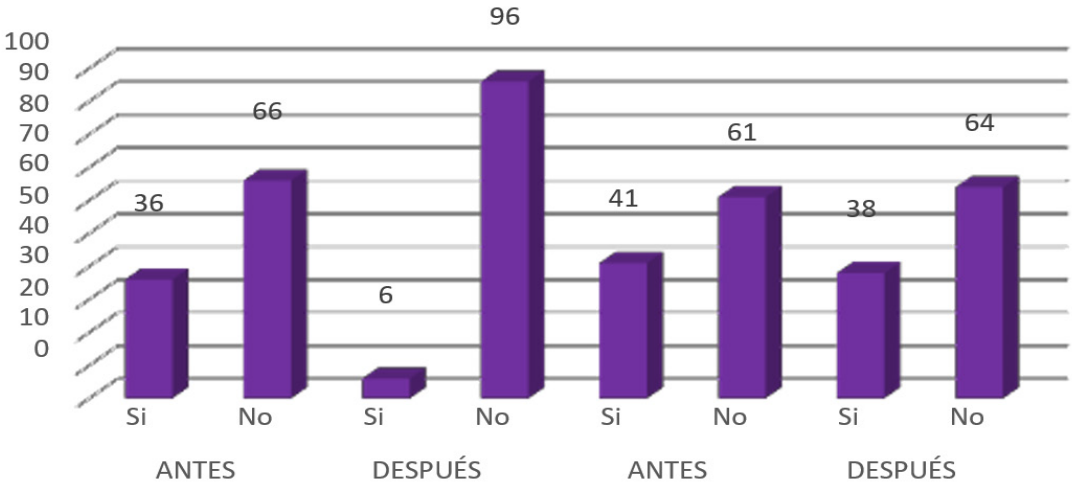

GRUPO EXPERIMENTO

GRUPO CONTROL

Fuente: Tabla 8

Gráfico 8. Prevalencia del consumo de alcohol en escolares de la IE. Santa Isabel, antes de la intervención educativa, distribuidos como Grupo Experimento y Grupo Control.

Tabla 9. Respuestas a la pregunta:¿Cuáles son las consecuencias que ocasiona el consumo de alcohol? en el Grupo Experimento y grupo control después de la intervención educativa.

\begin{tabular}{lccccc}
\hline \multirow{2}{*}{ CONSECUENCIAS } & \multicolumn{2}{c}{ GRUPO EXPERIMENTO } & \multicolumn{2}{c}{ GRUPO CONTROL } \\
\cline { 2 - 3 } \cline { 5 - 6 } & $\mathbf{n}$ & $\mathbf{\%}$ & & $\mathbf{n}$ & $\mathbf{\%}$ \\
\hline Afecta la capacidad de racionamiento & 18 & 50 & & 1 & 2 \\
Daño permanente de la memoria & 7 & 19 & & 1 & 2 \\
Relaciones sexuales de alto riesgo & 6 & 17 & & 0 & 0 \\
Problemas en sus relaciones interpersonales & 3 & 8 & & 1 & 2 \\
No responde & 2 & 6 & & 38 & 93 \\
TOTAL & 36 & 100 & & 41 & 100 \\
\hline
\end{tabular}

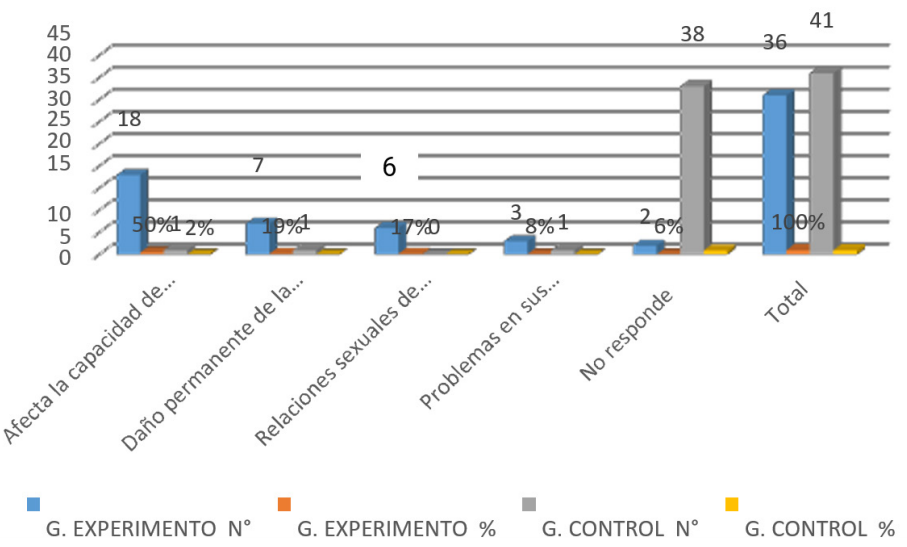

Gráfico 9. Respuestas a la pregunta: ¿Cuáles son las consecuencias que ocasiona el consumo de alcohol? en el Grupo Experimento y grupo control, después de la intervención educativa. 
Tabla 10. Respuestas a la pregunta: ¿Cuáles son los malestares que produce el consumo de alcohol en el organismo? en el Grupo Experimento y grupo control después de la intervención educativa.

\begin{tabular}{lcccccc}
\hline \multirow{2}{*}{ MALESTARES } & \multicolumn{2}{c}{ GRUPO EXPERIMENTO } & & \multicolumn{2}{c}{ GRUPO CONTROL } \\
\cline { 2 - 3 } \cline { 6 - 6 } & $\mathbf{n}$ & $\mathbf{\%}$ & & $\mathbf{n}$ & $\mathbf{\%}$ \\
\hline Alegria, tristeza y llanto & 21 & 58 & & 3 & 7 \\
Deseos de orinar & 14 & 39 & & 1 & 2 \\
No responde & 1 & 3 & & 37 & 90 \\
TOTAL & 36 & 100 & & 41 & 100 \\
\hline
\end{tabular}

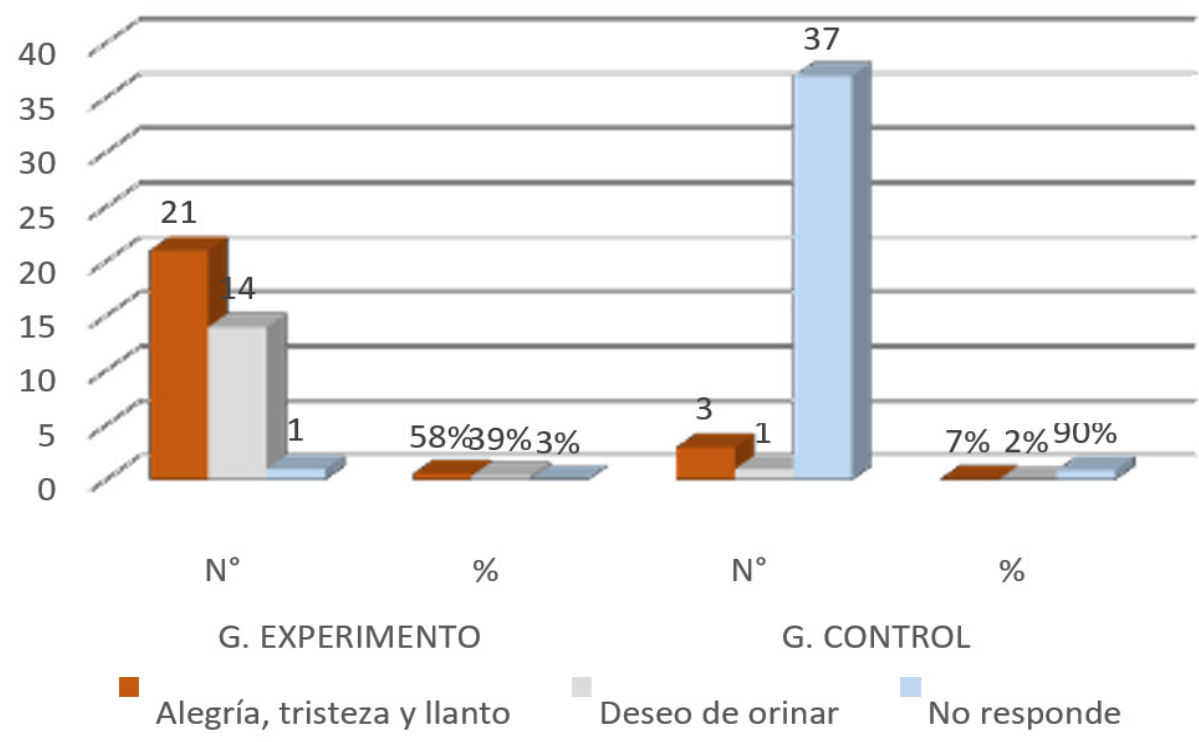

Fuente: Tabla 10

Gráfico 10. Respuestas a la pregunta:¿Cuáles son los malestares que produce el consumo de alcohol en el organismo? en el Grupo Experimento y grupo control después de la intervención educativa.

Tabla 11. Respuestas a la pregunta:¿Qué opinión te merece el consumo de alcohol? en el grupo experimento y grupo control después de la intervención educativa.

\begin{tabular}{lccccc}
\hline \multirow{2}{*}{ OPINION } & \multicolumn{2}{c}{ GRUPO EXPERIMENTO } & & \multicolumn{2}{c}{ GRUPO CONTROL } \\
\cline { 2 - 3 } \cline { 5 - 6 } & $\mathbf{n}$ & $\mathbf{\%}$ & & $\mathbf{n}$ & $\mathbf{\%}$ \\
\hline Malo & 25 & 69 & & 2 & 5 \\
Bueno & 3 & 8 & & 11 & 27 \\
No se debe tomar & 6 & 17 & & 0 & 0 \\
Es una droga & 2 & 6 & & 0 & 0 \\
No responde & 0 & 0 & & 28 & 68 \\
TOTAL & 36 & 100 & & 41 & 100 \\
\hline
\end{tabular}

así como daño permanente a la memoria y un $93 \%$ no respondieron.

En la tabla 10 y gráfico 10 , se puede apreciar que un $58 \%$ de los escolares que recibieron la intervención educativa respondieron que el consumo de alcohol le produce alegría, tristeza y llanto mientras que en el grupo control respondieron en 7\%, así también en el GE un 39\% respondieron deseo de orinar y en el GC un $2 \%$ respondieron, también un $3 \%$ no respondieron 


\section{INVESTIGACIÓN ORIGINALIORIGINAL RESEARCH}

Junchaya Yllescas $V . y$ col.

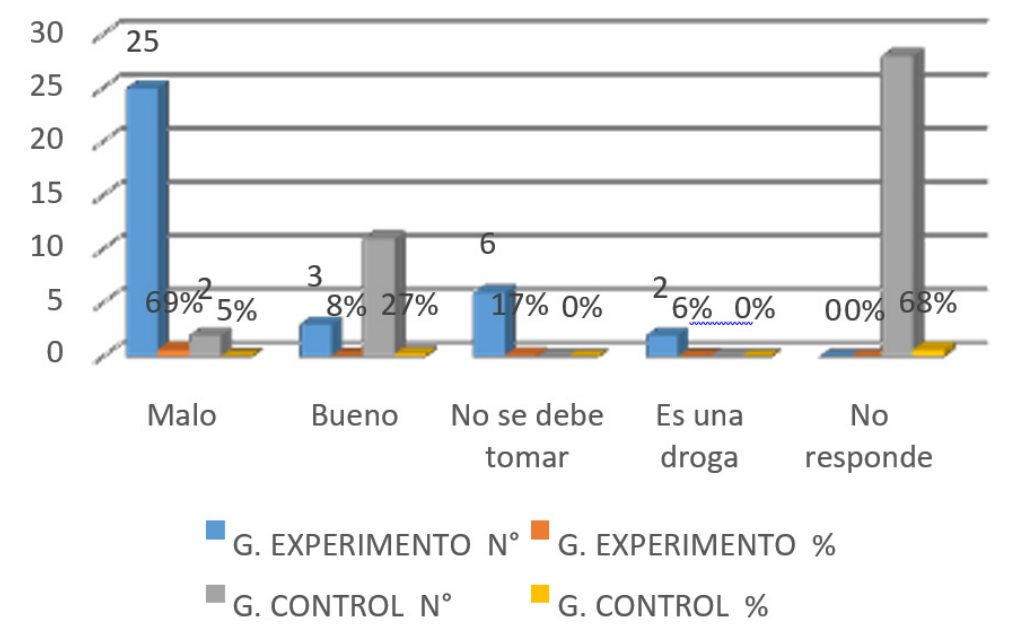

Gráfico 11. Respuestas a la pregunta: ¿Qué opinión te merece el consumo de alcohol? en el grupo experimento y grupo control después de la intervención educativa.

en el GE, mientras que en el GC lo hicieron en $90 \%$. En la tabla 11 y gráfico 11, sobre la opinión del consumo de alcohol en el GE respondieron en $69 \%$ como malo, un $8 \%$ como bueno y un $17 \%$ que no se debe tomar; en cambio en el grupo control un $27 \%$ respondieron como bueno, un 5\% como malo y un 68 $\%$ no responde.

\section{DISCUSIÓN}

Con respecto a la prevalencia del consumo de alcohol en escolares de la IE. Santa Isabel antes de la intervención educativa, se cuenta que un $38 \%$ de los escolares consumieron alcohol, lo que se corrobora con el estudio de Siveria et al., encontrándose que predominó el sexo masculino en un $73,47 \%$ y el grupo de edades entre $28-38$ años $(42,86 \%)$, prevaleciendo el nivel de escolaridad secundario en el 45,92\% (5), de igual forma con la investigación de Musayón et al., realizado como un estudio descriptivo - transversal, encontrándose como resultados que un $26,4 \%$ de escolares consumió alcohol en el último año (6), así también el trabajo de Salazar et al., quienes hallaron que el consumo de alcohol fue de $42,2 \%$, en escolares (7).

Así también la frecuencia del consumo de alcohol en escolares de la IE. Santa Isabel, se aprecia que lo hacen más de 2 veces al mes en $51 \%$, lo que guarda cierta similitud con el trabajo de López et al., en el cual se encontró que la frecuencia de consumo de las bebidas alcohólicas es de por lo menos 1 vez al mes en un 42,52\% (418/983), seguido en porcentaje por $1 \mathrm{vez}$ por semana que representa el 18,5\% (182/983) (8).

Además, en cuanto a los factores de riesgo personales se cuenta que el $35 \%$ de los escolares respondieron que consumen alcohol por diversión, seguido un $22 \%$, porque les gusta, lo que difiere con el trabajo de López et al., quienes resaltan, que la prevalencia del consumo de bebidas alcohólicas en colegios Asunción, es porque les gusta en el $60,1 \%$ y el $16,6 \%$ porque lo consumen los amigos (8).

En cuanto a los factores familiares que conllevan al consumo de bebidas alcohólicas en escolares de la IE Santa Isabel, se presenta que el 36\% de los escolares, quienes sostienen que consumen alcohol porque han faltado con claridad en las reglas que comunican los padres, seguido de discusiones entre padres y porque algún miembro de la familia ha consumido alcohol, lo que se contrapone con el trabajo de Salazar et al., quienes encontraron que los factores psicosociales más asociados al problema de consumo de drogas fue el distrés psicológico severo, además se encontró asociación entre el pensamiento problemático severo y el abuso físico con dicho problema (7).

De igual forma, en esta investigación se hallaron los factores amicales que ocasionan el consumo de alcohol, apreciándose que un $79 \%$ de los escolares sostienen que los factores que han ocasionado el consumo de alcohol es que algún miembro de la familia ha consumido alcohol, o porque algún amigo le 
ha regalado el alcohol, lo que guarda cierta semejanza con el trabajo de Fernández, quien demostró que se ven influenciados por la sociedad para el consumo de alcohol (9), de igual forma con el trabajo de Musayón et al., quienes encontraron como resultado que los factores fueron para el consumo de alcohol, que algún familiar hubiera consumido alcohol o que alguno de los amigos consumen alcohol (6).

Del mismo modo, en esta investigación se encontró que un $63 \%$, sostienen que consumen alcohol para sentirse bien, lo que se corrobora con el estudio de Musayón et al., quienes demuestran que se ha consumido alcohol para sentirse bien $(\mathrm{p}=0,000)(6)$.

Al medir el efecto de la intervención educativa, se puede apreciar que existe una diferencia significativa en las respuestas sobre el consumo de bebidas alcohólicas, es decir antes de la intervención educativa los estudiantes del GE, respondieron que consumen alcohol en 66, mientras que después de la intervención educativa se redujo a 6 , datos que no fueron favorables para el GC. Sin embargo, se puede apreciar que en el GE la intervención farmacéutica favoreció los resultados mediante la aplicación de 7 sesiones educativas: como introducción al consumo de bebidas alcohólicas, mi familia y yo, factores o causas del consumo de alcohol en adolescentes, consecuencias del consumo de alcohol en adolescentes y tratamiento del consumo de alcohol en adolescentes, resultados que se corroboran con el trabajo de Quispe, en el que se aprecia que existe una diferencia significativa en las respuestas de los escolares antes y después de la Intervención educativa, lo que indica que los talleres educativos influyen significativamente en la percepción del consumo de alcohol en los escolares del presente estudio (10).

Analizando sobre el efecto de la intervención educativa y en cuanto a las respuestas de los estudiantes sobre las consecuencias que produce el alcohol, se puede apreciar que la mitad de los estudiantes del GE, responden que el consumo de alcohol afecta la capacidad de razonamiento y juicio, daño permanente de la memoria, relaciones sociales de alto riesgo, a diferencia que en el grupo control solo un $2 \%$ respondieron que el consumo de alcohol afecta la capacidad de razonamiento y juicio.

Es oportuno mencionar que un $58 \%$ de los escolares que recibieron la intervención educativa respondieron correctamente que el consumo de alcohol le produce alegría, tristeza y llanto mientras que en el grupo control solo respondieron en 7\%. Cabe concluir que el $69 \%$ de los estudiantes del GE, respondieron que el consumo de alcohol es malo, mientras que en el GC solo lo hicieron en $27 \%$. Lo que de cierta manera se corrobora con el trabajo de Quispe, quien encontró que el $94,7 \%$ de la muestra no presenta tener problemas con el alcohol (10). Sin embargo, resulta preocupante lo encontrado en el grupo control de esta investigación que no recibió la intervención educativa y se contrapone al trabajo de Colqui et al., en el que se observa que los adolescentes que han consumido bebidas alcohólicas en los últimos 30 días fueron en $24 \%$ (11).

Es justo decir, que la Organización Mundial de la Salud (OMS), define a la adolescencia, como el periodo en el cual la persona progresa hasta la maduración sexual considerándolo desde los 10 a 19 años de edad (12), y de acuerdo a esta definición para lograr un desarrollo saludable los adolescentes necesitan primero haber tenido una infancia saludable, en un ambiente seguro, situación que lamentablemente no se produce actualmente en los hogares, ocasionándose situaciones que entran en juego el grado de exposición a las bebidas alcohólicas, las circunstancias y el contexto en que se produce la ingestión de esta sustancia; existiendo evidencias de que los jóvenes están comenzando a beber a edades cada vez más tempranas en el Perú, por lo cual actualmente, se deben adoptar medidas que permitan regular, controlar y eliminar esta sustancia lícita de mayor publicidad en adolescentes, haciéndose cumplir la Normatividad Nacional con el marco necesario para la prevención en protección de la infancia. Nos parece importante instar a seguir investigando en esta área, abarcando más estudiantes e incluyendo a menores de trece años, así como iniciar tareas educativas en toda esta población.

\section{CONCLUSIONES}

Se puede concluir que el consumo de bebidas alcohólicas en adolescentes constituye un problema de salud en el territorio, corresponde a la familia, la escuela y a la comunidad, contribuir mediante una estrategia de intervención educativa a la reducción del consumo es importante en el grupo experimento a diferencia del grupo control.

Los factores de riesgo importantes de los adolescentes para el consumo de bebidas alcohólicas fueron los de tipo personal porque consumen por diversión y les gusta, familiares, porque faltan a reglas y discusiones de padres, amicales, cuando algún 
miembro ha consumido alcohol o le obsequian alcohol y sociales cuando no hay supervisión de personas mayores en las fiestas.

Es importante considerar que los adolescentes al término de la intervención educativa consideran que el consumo de alcohol afecta la capacidad de razonamiento y juicio a diferencia que el grupo control desconoce la información.

Estos hallazgos inducen a realizar actividades educativas utilizando dinámicas familiares y otras técnicas con la participación de grupos de relación y acciones intersectoriales que incluyan esta vez a toda la familia.

\section{Correspondencia}

Mg. Vilma Amparo Junchaya Yllescas

Correo electrónico: ajunchaya@uroosevelt.edu.pe

\section{REFERENCIAS BIBLIOGRÁFICAS}

1. Zeigler DW, Wang CC, Yoast RA, et al. The neurocognitive effects of alcohol on adolescents and college students. Preventive Medicine. 2005; 40:23-32.

2. Sánchez R. Publicidad de bebidas alcohólicas. Algunas razones para establecer unos límites. Adicciones. 2002; 14 (Supl. 1): 133-40.

3. World Health Organization. The global trategy to reduce the harmful use of alcohol. Geneva: World Health Organization; 2010.

4. Obot IS, Saxena S. (eds.). Substance use among young people in urban environments. Geneva:World Health Organization; 2005.

5. Siveria D, Serrano Y, Miranda T, Agramonte M, González JC. Intervención educativa sobre consumo excesivo de alcohol. Revista Electrónica de PortalesMedicos.com; 2016; 1: 0-0. (Fecha de acceso 13 de Julio del 2017) Disponible en: https:// www.revista-portalesmedicos.com/revista-medica/ consumo-excesivo-de-alcohol/.
6. Musayón Y, Torres O, Sánchez E, Chávez E. Factores de riesgo del consumo de bebidas alcohólicas en escolares de educación secundaria. Investigación y Educación en Enfermería. 2005; 23(1): 54-67.

7. Salazar E, Ugarte M, Vásquez L, Loaiza J. Consumo de alcohol y drogas y factores psicosociales asociados en adolescentes de Lima. An Fac Med. 2004. 65(3): 179-187.

8. López L, Gonzales L, Pacheco L. Prevalencia del consumo de bebidas alcohólicas en colegios de estudiantes de colegios de Asunción. Anales de la Facultad de Ciencias Médicas. 2005; 38(3): 82-87. (Fecha de acceso 25 de Julio del 2018) Disponible en URL: http://revistascientificas.una.py/index.php/ $\mathrm{RP} /$ article/ viewFile/545/452

9. Fernández S. Evaluación de la efectividad de los programas escolares de prevención del consumo de tabaco y alcohol y cannabis ¿Qué nos dicen los Metaanalisis? Revista Española de Salud Pública. 2002; 76(3): 175-187. (Fecha de acceso 25 de Julio del 2018) Disponible en URL:https://www.redalyc. org/pdf/170/17076302.pdf

10. Quispe M. Influencia de la intervención educativa en la percepción del consumo de alcohol en escolares de 12 a 17 Años de la I.E. Mixta Cecilia Túpac Amaru del Cusco 2013. Tesis de Grado. Lima, Perú: Universidad Nacional de San Antonio Abad del Cusco; 2017.

11. Colqui M, Ortega A, Salas M, Vaiz R. Prevalencia de consumo de alcohol en adolescentes de las Instituciones Educativas Estatales, Distrito de Ventanilla. Rev enferm Herediana. 2015;8(2):3-10.

12. Organización Mundial de la Salud. Desarrollo en la adolescencia. Ginebra: Organización Mundial de la Salud; 2015.

Recibido: 01/11/2019

Aceptado: 20/03/2020 